\title{
Nanoreceptors promote mutant p53 protein degradation by mimicking selective autophagy receptors
}

Long-ping Wen ( $\sim$ lpwen@ustc.edu.cn )

Department of Cardiology, Guangdong Provincial People's Hospital \& Guangdong Academy of Medical Sciences

\section{Xiaowan Huang}

School of Medicine, South China University of Technology

\section{Ziyang Cao}

Department of General Surgery, Guangzhou First People's Hospital, South China University of Technology

\section{Tao Ding}

School of Medicine, South China University of Technology

\section{Jieying Qian}

South China University of Technology

Hao Zhang

South China University of Technology

\section{Suqin Zhong}

South China University of Technology

\section{Xiaoli Wang}

School of Medicine, South China University of Technology

\section{Guangyu Yao}

Breast Center, Department of General Surgery, Nanfang Hospital, Southern Medical University

\section{Xian-Zhu Yang}

South China University of Technology https://orcid.org/0000-0002-1006-0950

\section{Yunjiao Zhang}

School of Medicine, South China University of Technology, Guangzhou, China

\section{Article}

Keywords: nanoreceptors, protein aggregates, selective autophagy, mutant p53, cancer therapy

Posted Date: November 30th, 2021

DOI: https://doi.org/10.21203/rs.3.rs-1039913/v1 
License: (c) (i) This work is licensed under a Creative Commons Attribution 4.0 International License. Read Full License 


\section{Abstract}

More than half of human malignant tumors harbor TP53 gene mutations, most of which are point mutations within the DNA-binding domain of TP53, resulting in mutant p53 (mutp53) protein stabilization and accumulation in the cell and enhanced tumor progression. Depletion of mutp53 through the autophagy or proteasome pathway is considered the most direct strategy to target mutp53 for tumor treatment. However, due to the lack of specific autophagy receptors and the insufficient level of autophagy in tumor cells, targeted degradation of mutp53 by nanomaterials via the autophagy pathway has not been reported. Here, we propose a type of "nanoreceptors" (denoted NRs) that mimics selective autophagy receptors and develop a new platform for targeted degradation of mutp53. The NRs specifically bind mutp53 in tumor cells via mutp53-binding peptide (MBP). In addition, the level of cell autophagy is greatly increased due to the incorporated of cationic lipid. As a result, the NRs effectively degrade mutp53 through the autophagy pathway with complete autophagic flux. The knockout of ATG5, an essential autophagy-related gene, significantly inhibited the NRs-induced degradation of mutp53, demonstrating the critical role of autophagy in this effect. Subsequently, the degradation of mutp53 by the NRs abrogated mutp53-conferred gain-of-function (GOF) phenotypes, including enhanced cell proliferation and cell migration and reduced sensitivity to cisplatin (CDDP). Last, Pt(IV)-loaded NRs (NRs/Pt, consisting of Pt(IV) prodrug encapsulated in the NRs) showed outstanding synergistic antitumor effects in an ES-2 ovarian cancer model and a patient-derived xenograft (PDX) ovarian cancer model. Collectively, our study suggests the use of NRs/Pt as a new biomimetic nanoplatform for regulating autophagy, providing new ideas for precise tumor treatments that target mutp53.

\section{Introduction}

The tumor suppressor p53, encoded by the TP53 gene, is an important tumor transcription factor that serves to maintain cell homeostasis by regulating cell cycle arrest and cell death in response to a variety of stress signals ${ }^{1-4}$. Mutations of TP53, the most commonly occurring gene alterations during tumorigenesis, have been observed in at least half of human cancers ${ }^{5,6}$. Large-scale analysis of human genomics showed that the frequency of p53 mutations is high in certain types of cancer, such as basal breast cancer (88\%), pancreatic cancer (41\%), lung squamous cell carcinoma (84\%), and high-grade serous ovarian cancer (as high as $96 \%)^{7,8}$. Among these mutations, the most common are missense mutations that involve a single amino acid change, including S241F, R248W, R273H, R282W, and R175H ${ }^{9}$, 10. In contrast to the wild-type p53 protein, missense mutant p53 (mutp53) proteins are stabilized and accumulate to high levels in the cell ${ }^{11}$. These mutants not only lead to cancer progression due to loss of wild-type p53 activity but also enhanced oncogenic functions (gain-of-function (GOF)), including enhanced tumorigenesis, invasion, metastasis and resistance to therapeutic drugs ${ }^{12,13}$.

Given its widespread presence in human cancers and key role in tumor progression, targeting GOF mutp53 is the aim of some attractive therapeutic strategies ${ }^{14,15}$. Among them, inducing mutp53 degradation is the most straightforward strategy for disease treatment in eukaryotes, but proteasomes do 
not efficiently degrade certain large proteins or aggregates due to the inability of these factors to enter the narrow cavity of the proteasome ${ }^{16}$. Macroautophagy (hereafter referred to as autophagy), an independent protein degradation pathway, involves the phagocytosis of long cyclins and protein aggregates in the cytoplasm into autophagosomes for subsequent lysosomal degradation ${ }^{17-19}$. An increasing number of studies have found that the process of autophagy is highly selective for cargoes in some cases due to regulation by autophagy receptors. Autophagy receptors are endowed with the ability to both recognize the ubiquitination signals of cargoes by binding the ubiquitin-binding domain (UBD) and interact with the ATG8/LC3/GABARAP protein on the autophagosome membrane, relying on their LC3-binding region (LIR), which is followed by the delivery of cytoplasmic cargo to the incipient autophagosome for engulfment and ultimately lysosomal degradation ${ }^{20-22}$. Notably, a number of specific autophagy receptors, including SQSTM1/p62, OPTN, NBR1, and NDP52, have been reported to mediate the degradation of huntingtin 23 , phosphorylated tau ${ }^{24}$ and Salmonella enterica ${ }^{25}$. Nevertheless, engineered to achieve the degradation of specific proteins by selective autophagy is unclear.

Based on the biological principles of selective autophagy, mutp53 aggregates can be engulfed into double-membrane autophagosomes associated with lipidated LC3 and degraded by autophagy. We therefore hypothesized that an appropriate autophagy receptor that interacts with both mutp53 and LC3 may enhance the recruitment of mutp53 aggregates into autophagosomes, elevating autophagy and subsequently facilitating mutp53 degradation. Thus, we designed a multifunctional engineered biomimetic nanoparticle that mimics autophagy receptors, simultaneously binding mutp53 and mediating mutp53 degradation by the autophagy pathway. Thus, we propose the design of multifunctional engineered "nanoreceptors" (NRs) formed by the self-assembly of maleimide-polyethylene glycol-polylactic acid (Mal-PEG-PLA) with incorporated 1,2-dioleoyl-3-trimethylammonium-propane (DOTAP, a cationic lipid) whose surface is further covalently modified with a mutp53-binding peptide $(\mathrm{MBP})^{26}$. On the one hand, MBP on the surface of the NRs targets and binds mutp53. On the other hand, cationic lipid elevate the level of autophagy in tumor cells by inducing increased autophagosome formation and play a role in bringing NRs-bound mutp53 into autophagosomes. Collectively, NRs function as mutp53-specific autophagy receptors and achieve efficient autophagic degradation of mutp53.

\section{Results And Discussion}

\section{Preparation of engineered biomimetic NRs that mimic the autophagy receptor}

With the above considerations in mind, we first assessed the potential role of cationic lipid (DOTAP) in autophagy-inducing activity in EGFP-LC3/MDA-MB-231 cells, a breast cancer cell line that stably expresses enhanced green fluorescent protein-tagged LC3 (EGFP-LC3) and p53-R280K mutant. Largely consistent with published reports ${ }^{27,28}$, DOTAP treatment induced a significant increase in punctate dot formation compared to that in untreated cells under fluorescence microscopy (Fig S1) and triggered the colocalization of these puncta with lysosomes (Fig S2), demonstrating the elevation of autophagy levels 
and autophagosome formation, respectively. Then, we rationally designed and prepared NRs (nanoreceptors, MBP-NPs/DOTAP) (Fig 1a), a type of engineered biomimetic nanoparticle with multiple functions that mimics autophagy receptors, binding mutp53 via MBP-specific recognition and inducing the autophagy-mediated degradation of mutp53 via DOTAP. Dynamic light scattering (DLS) and transmission electron microscopy (TEM) analyses indicated that NRs, dNRs (NPs/DOTAP, without covalent modification with MBP as one negative control), and mNRs (MBP-NPs, without incorporated DOTAP as another negative control) had a spherical morphology with similar sizes of approximately 100 nm (Fig 1b and Fig S3) and good stability in 10\% FBS for $24 \mathrm{~h}$ (Fig S4). However, the zeta potentials of dNRs and NRs were positive $(\sim+15 \mathrm{mV})$ due to the incorporated DOTAP, while the mNRs had a negative charge of approximately $-7.5 \mathrm{mV}$ due to the absence of DOTAP (Fig S5). Next, circular dichroism (CD) spectra were obtained to investigate the interaction between the mutp53 protein and free MBP or two NPs. As shown in Fig 1c, in both the absence and presence of dNRs, the mutp53 protein spectrum contained two negative peaks at $208 \mathrm{~nm}$ and $222 \mathrm{~nm}$, corresponding to the helical character of a protein. In contrast, the spectrum of mutp53 incubated with NRs exhibited a minimum peak at $231 \mathrm{~nm}$, revealing that the secondary structure of the mutp53 protein had noticeably transformed and indicating obvious binding with NRs. Notably, free MBP did not affect the secondary structure of mutp53, which may be due to the low molecular weight of the peptide. To gain further proof of the interaction between mutp53 and the NRs, we conducted surface plasmon resonance (SPR). First, NRs were immobilized onto a chip through amide coupling utilizing the primary amine in the MBP. Then, mutp53 at five concentrations ( $2500 \mathrm{nM}, 500 \mathrm{nM}, 100 \mathrm{nM}, 20 \mathrm{nM}$ and $4 \mathrm{nM}$ ) in PBS was injected onto the channel, and the response units were measured over a single cycle. As shown in Fig $1 \mathrm{~d}$, the binding isotherms between mutp53 and the NRs fit a standard 1:1 binding model, and further single-cycle kinetic measurement of the interactions between mutp53 and NRs determined a KD value of $1.40^{\prime} 10^{-9}$, indicating that the NRs can effectively bind mutp53 at various protein concentrations. In comparison, the binding efficiency between dNRs (without MBP modification) and mutp53 was much lower than that between NRs and mutp53 (Fig S6). This finding indicated that the NRs can effectively bind mutp53 through specific high-affinity interactions.

Furthermore, binding of mutp53 to the NRs surface via MBP was demonstrated by stochastic optical reconstruction microscopy (STORM). Each component of the NRs (the NP and MBP) and mutp53 were labeled with different fluorophores and independently imaged in their respective channels. As shown in Fig 1e, the MBP (green) was observed to surround the NP core (red), which demonstrated that the MBP had been successfully conjugated onto the NP surface to obtain the NRs. Furthermore, when mutp53 was added to the NRs solution, binding took place, and mutp53 (blue) was primarily located on the external surface of the NRs, indicating that the NRs could effectively bind mutp53 through high-affinity interactions between the MBP and mutp53 (Fig 1f).

Taken together, the above results demonstrated that the NRs could bind mutp53 with specific high-affinity interactions, providing a preliminary basis for its ability to mimic autophagy receptors to capture mutp53 in vivo. 


\section{The NRs induced the rapid degradation of mutp53}

As described above, degradation of the mutp53 protein requires two key processes. One is to elevate the level of autophagy to ensure enough autophagosomes, and the other is to enhance the recruitment of mutp53 into autophagosomes, facilitating its degradation. To determine the appropriate concentration of DOTAP, NRs into which different concentrations of DOTAP had been incorporated were prepared, and whether DOTAP concentration affected mutp53 stability was evaluated in ES-2 cells, which harbor the S241F mutation in the TP53 gene. Our results showed that mutp53 levels decreased upon the use of NRs containing $5 \%$ DOTAP at a dose of $500 \mu \mathrm{g} / \mathrm{ml}$ NRs and gradually decreased as the DOTAP concentration increased (Fig 2a). We also explored the toxicity to normal cells of NRs in which DOTAP at different concentrations was incorporated. The results of an MTT assay implied that NRs containing 40\% DOTAP exhibited increased toxicity in the normal HEK 293 cell line due to the positive charge of DOTAP (Fig S7). Therefore, NRs containing 20\% DOTAP were selected as the optimum formulation and used in subsequent experiments with NRs. Next, we exhaustively investigated the ability of free peptide, mNRs, dNRs and NRs to deplete mutp53 in ES-2 ovarian cancer cells. Immunofluorescence analysis showed that NRs significantly reduced mutp53 levels compared to those in the other control groups (Fig 2b). The reduced mutp53 protein level was also verified by Western blotting (Fig 2c). In addition, we assessed autophagy-inducing activity of the different treatments in ES-2 cells. The conversion of microtubuleassociated protein light chain 3 (LC3), indicating conversion of the endogenous soluble LC3-I protein to lipid-bound LC3-II after attachment to the autophagosome membranes, was significantly elevated after NRs treatment (Fig 2c). To explore the mechanism of mutp53 depletion by the NRs, we first assessed whether NRs treatment would affect the mRNA level of mutp53 in ES-2 cells (Fig 2d). Cotreatment with the protein synthesis inhibitor cycloheximide (CHX) accelerated the reduction in mutp53 induced by NRs (Fig 2e), indicating that the effect of NRs on mutp53 was due to degradation instead of reduced protein synthesis. To determine whether the effect of NRs on mutp53 was specific, we tested the effect of NRs on ES-2 (ovarian cancer cells expressing p53-S241F mutant), MDA-MB-231 (breast cancer cells expressing p53-R280K mutant), MIA PaCa2 (colorectal cancer cells expressing p53-R248W mutant) and SK-BR3 (breast cancer cells expressing p53-R175H mutant). Similar to the results in the ES-2 cell line, the NRs also dramatically degraded mutp53 and induced the conversion of LC3-II in a dose-dependent manner in three mutp53 cell lines. (Fig 2f, S8 and S9, respectively). Consistent results were also observed using ectopically expressed p53-S241F, p53-R248W and p53-R175H in p53-null H1299 cells upon NRs treatment, further suggesting that the NRs broadly deplete multiple p53 mutants (Fig 2g). Taken together, the above results demonstrated that NRs could degrade mutp53 in a variety of cell lines expressing mutp53.

\section{NRs degrade mutp53 in an autophagy-dependent manner.}

To evaluate the mechanism by which NRs elicited mutp53 degradation, we first assessed whether cellular internalization was necessary for this process. Genistein, an inhibitor of caveolae-dependent endocytosis, effectively inhibited mutp53 degradation and LC3-II conversion induced by NRs (Fig S10), suggesting that it was necessary for NRs to enter the cell before degrading mutp53. There are two main ways to degrade 
mutant proteins in the cell, namely, the proteasome and autophagy pathways. The autophagy inhibitor wortmannin effectively inhibited mutp53 degradation caused by NRs, but the proteasome inhibitor MG132 failed to do so (Fig 3a). This revealed that the NRs may degrade mutp53 through the autophagy pathway. To verify the role of autophagy in mutp53 degradation, we knocked out ATG5, an essential autophagy-related gene, in ES-2 cells with lentiviral shRNA interference technology. Knockout of ATG5 resulted in a decrease in LC3-II conversion, indicative of a decrease in autophagy levels, in ES-2 cells treated with NRs. ATG5 knockout significantly inhibited the degradation of mutp53 caused by NRs, which indicated that this process was autophagy-dependent (Fig 3b). TEM also showed a significant increase in autophagosomes after $12 \mathrm{~h}$ of NRs treatment in ES-2 cells, and the most highly magnified image clearly showed the presence of autophagic vesicles containing partially degraded cytoplasmic material and increased electrons (Fig 3c). Furthermore, consistent with the results showing that autophagy was induced and autophagosomes were produced after treatment with DOTAP alone (Fig S2), we synthesized mNRs and NRs with encapsulated DiD, a fluorescent dye, and found that the number of LC3 puncta significantly increased, suggesting increased autophagosomes, and that the puncta colocalized with NRs and LysoTracker Red, a selective dye that stains lysosomes, in the NRs-treated cells compared with the mNRs-treated cells (Fig 3d).

These results fully proved that the NRs could induce autophagy by triggering the accumulation of autophagosomes. Autophagy is a dynamic process, and the increase in autophagosome accumulation could have resulted from increased autophagosome formation or blocked downstream degradation of autophagosomes. To determine the mechanism utilized by the NRs, we measured LC3-II conversion after cotreatment with the autophagy inhibitors 3-MA and bafilomycin A1, which inhibit autophagosome formation and fusion of autophagosomes and lysosomes, respectively. NRs treatment led to an increase in LC3-II conversion, and 3-MA inhibited the NRs-induced increase in LC3-II conversion, while bafilomycin A1 promoted it (Fig $3 \mathrm{e}$ ). These results indicated that the NRs-treated cells exhibited normal autophagic flux and enhanced autophagosome formation. Meanwhile, cotreatment with 3-MA and bafilomycin A1 also inhibited the degradation of mutp53 triggered by NRs, further illustrating that the NRs degraded mutp53 through the autophagy pathway (Fig 3e). The immunofluorescence results showed that the level of mutp53 was decreased and the level of LC3 was increased after treatment with NRs compared to the control group. In addition, mutp53 colocalized with LC3 proteins and NPs in the cells, suggesting the importance of autophagy when NRs degrade mutp53 (Fig S11).

We also detected changes in the content of SQSTM1/p62, a common endogenous autophagy substrate, to test the level of autophagy. The level of SQSTM1/p62 significantly decreased after NRs treatment in a time-dependent manner. Similarly, the conversion of LC3-Il gradually increased when NRs were applied (Fig 3f). To obtain further evidence that the NRs induced complete autophagy with normal cargo degradation, we established MDA-MB-231 cells stably expressing EGFP-mCherry-LC3, an LC3 fusion protein that exhibited both green (EGFP) and red (mCherry) fluorescence. The green fluorescence from EGFP is easily quenched in an acidic environment, such as the environment in autolysosomes, while the red fluorescence from mCherry is relatively stable. Therefore, the fusion of autophagosomes and lysosomes is indicated by a relative increase in red fluorescence and a decrease in green fluorescence. 
This was indeed confirmed after NRs treatment but not in the control group (Fig 3g). In addition, the NRs enhanced the ubiquitination level of mutp53, including its K63 polyubiquitination, in ES-2 cells (Fig 3h), and the protein ubiquitination inhibitor PYR-41 effectively inhibited the degradation of mutp53 induced by NRs but did not affect the level of autophagy in the cell (Fig 3i).

These results indicate that the NRs degrade mutp53 by selective autophagy in a ubiquitination-dependent manner, increase the production of autophagosomes and trigger complete autophagic flux.

\section{Degradation of mutp53 by NRs abrogated mutp53-conferred GOF phenotypes}

Missense mutation of the TP53 gene leads to dysfunctional mutp53 proteins that are stabilized and accumulate in the tumor, exhibiting oncogenic GOF phenotypes to promote tumor cell growth, survival and migration. Therefore, the degradation of mutp53 by NRs would be expected to show stronger toxicity in p53 mutant cells than in normal cells. Indeed, NRs treatment obviously reduced cell viability in four p53 mutant cell lines (MIA PaCa-2, SK-BR-3, MDA-MB-231 and ES-2 cells) and even decreased viability by 75\% in ES-2 cells but had almost no effect on normal HEK 293T cells or HUVECs (Fig 4a). In addition, we established mutp53-knockout ES-2 cells by CRISPR/Cas9 technology (Fig S12). mutp53 knockout significantly reduced cell proliferation, but NRs treatment did not show further toxicity in these cells, which indicated that the NRs-induced toxicity observed in ES-2 cells was mediated by mutp53 (Fig 4b). Additionally, sphere formation and clone formation assays were used to evaluate the proliferative ability of the ES-2 cells. In comparison to PBS-, MBP-, mNRs- and dNRs-treated cells, NRs-treated cells showed an efficient reduction in sphere formation and clonal formation (Fig $4 \mathrm{c}$ and Fig $4 \mathrm{~d}$ ). The results of a Transwell assay showed that the migration of ES-2 cells treated with NRs was also significantly inhibited (Fig 4e). We further detected cell death by propidium iodide (PI) staining in ES-2 cells, and the NRs caused an increase in red fluorescence and significant increase in cell death compared to those of other groups of cells (Fig 4f). In addition, mutations in the p53 protein make it more difficult for chemotherapy to kill cancer cells, which led us to consider the potential of NRs-induced degradation of mutp53 to enhance sensitivity to chemotherapy drugs. Therefore, we tested the cell viability of ES-2 tumor cells treated with NRs alone or NRs combined with cisplatin (CDDP). Cotreatment with NRs and CDDP effectively enhanced the death of ES-2 cells compared with that upon treatment with NRs or CDDP alone (Fig 4g). Thus, we concluded that the NRs killed p53-mutated tumor cells by eliminating mutp53-conferred GOF phenotypes.

\section{The NRs significantly enhanced chemotherapeutic efficacy in the ES-2 ovarian cancer model and the p53-mutated patient-derived xenograft ovarian cancer model}

To further assess the antitumor effect of the NRs, we evaluated the ability of the NRs to deliver CDDP, a common clinical chemotherapeutic drug for ovarian cancer, and the synergistic antitumor effects of CDDP and the NRs, which may act as a chemosensitizer for the treatment of p53-mutant cancers. First, we used encapsulated platinum prodrug (IV) in the NRs through nanoprecipitation and obtained NRs/Pt(IV) (Fig 5a). To investigate the accumulation and retention of NRs/Pt in the tumor site, ES-2 tumor-bearing mice were intravenously (i.v.) administered DiD-labeled NRs/Pt and then monitored by a fluorescence in vivo imaging system (IVIS). As shown in Fig 5b, the mice exhibited the greatest 
fluorescence signals at the tumor site at $8 \mathrm{~h}$ postinjection, which could be due to the enhanced permeability and retention of the NRs/Pt. Moreover, ex vivo imaging at $48 \mathrm{~h}$ postinjection clearly revealed intense fluorescence signals in the tumor tissues (Fig 5c). Quantitative region-of-interest (ROI) analysis showed that the NRs/Pt were mostly distributed in the tumor site, followed by the lung, liver, kidney, spleen and heart, after i.v. administration (Fig 5d). Hematoxylin and eosin (H\&E) staining showed that none of the major organs (liver, spleen, kidney, lung, and heart) of the mice showed obvious tissue damage after treatment with PBS, dNRs, dNRs/Pt, NRs or NRs/Pt (Fig S13). Assessment of serum biochemical markers for liver (alanine aminotransferase and aspartate transaminase) and kidney (urea nitrogen and creatinine) toxicity also revealed no statistically significant difference in any of these markers upon nanoparticles treatment (Fig S14).

To evaluate the in vivo antitumor effect of NRs and their synergistic antitumor effects with CDDP, we constructed a subcutaneous xenograft nude mouse model from ES-2 cells. Seven groups, the PBS, dNRs, CDDP, dNRs/Pt, NRs, NRs+CDDP and NRs/Pt groups, were analyzed for the comparative study. All groups underwent a 13-day treatment regimen by tail vein every two days starting when the tumor volumes reached approximately $100 \mathrm{~mm}^{3}$. There was no significant difference in weight during the treatment period among the different treatment groups, although the mice in the CDDP and NRs+CDDP groups showed lower weights, which may be due to the toxicity of CDDP (Fig S15). Compared to the PBS, dNRs, CDDP and dNRs/Pt groups, the NRs group and NRs+CDDP group showed significantly inhibited tumor growth, and the NRs/Pt group showed a further reduction in tumor volume (Fig $5 \mathrm{e}$ ). In addition, the tumor weights were consistent with the tumor volume data. Treatment with the NRs and combination treatment with the chemotherapy drug CDDP effectively inhibited tumor growth, and NRs/Pt further enhanced tumor weight (Fig $5 f$ and Fig $5 \mathrm{~g}$ ). In agreement with the results obtained in the cultured cells, the experimental groups treated with NRs (including NRs, NRs+CDDP and NRs/Pt) exhibited effectively enhanced autophagy levels and decreased mutp53 levels (by Western blotting, immunohistochemistry and immunofluorescence) in tumor tissues (Fig 5h, 5i and S16, respectively). Finally, TUNEL assays also confirmed the antitumor efficacy of $\mathrm{NRs} / \mathrm{Pt}$, which increased cell apoptosis compared to that upon treatment with NRs, dNRs or CDDP alone (Fig S17).

To initially understand the potential clinical utility of NRs, we further established an orthotopically implanted p53 mutant $\left(\mathrm{P}_{2} 2 \mathrm{R}^{+/+}, \mathrm{C}_{141} \mathrm{Y}^{+/+}\right.$and $\left.\mathrm{L} 350 \mathrm{P}^{+/}\right)$patient-derived xenograft (PDX) ovarian cancer model in NOD/SCID mice. Mice were injected with PBS, CDDP, NRs or NRs/Pt by tail vein over every two days over a 13-day treatment regimen, starting when the tumor volumes reached approximately $100 \mathrm{~mm}^{3}$ (Fig 6a). Similarly, no significant difference in body weight change was observed among the four groups (Fig 6b). The NRs effectively reduced the increase in tumor volume, and the NRs/Pt further enhanced the tumor-suppressive effect of the NRs (Fig 6c). Consistently, the NRs alone significantly reduced tumor weight, and the NRs/Pt elicited a further significant reduction in tumor weight (Fig 6d, with the tumor photographs provided in Fig S18). More importantly, NRs/Pt and NRs to a lesser extent also enhanced autophagy (Fig 6e), reduced mutp53 levels (Fig 6e and Fig 6f), and enhanced cell apoptosis in the tumor tissue (Fig 6f). 


\section{Discussion}

Currently, 11 million patients worldwide live with tumors expressing highly stable mutp53 proteins ${ }^{29}$. Therefore, it is expected that targeting mutp53 for degradation will selectively target tumor cells with minimal impact on healthy tissues, thereby providing precise treatment for these patients. In our work, we have designed multifunctional engineered "nanoreceptors" (NRs) to mimic key autophagy receptors to degrade mutp53 in tumor cells. These NRs are composed of nanoparticle carriers modified with multifunctional polypeptides and the cationic lipid DOTAP, which can recognize and transport mutp53 to the autophagosome for degradation. We demonstrated that the NRs could effectively degrade mutp53 proteins in an autophagy- and ubiquitination-dependent manner in ES-2 cells, which harbored the p53 S241F mutant. Degradation of mutp53 by the NRs effectively abrogated mutp53-conferred GOF phenotypes, with exacerbated effects observed in mutp53-expressing tumor cells, including reduced cell proliferation and migration and enhanced cell death and sensitivity to the chemotherapy drug CDDP. Then, we obtained NRs/Pt(IV) by encapsulating platinum prodrugs (IV) in NRs and evaluated their biosafety and potential for the treatment of p53 mutant cancers. The NRs/Pt significantly enhanced the therapeutic effect of CDDP in an ES-2 ovarian cancer model and a PDX ovarian cancer model.

The accumulation of mutant proteins is a major cause of many diseases and decreasing the levels of these proteins can be useful for the treatment of these diseases. These mutp53 proteins are accompanied by many other pathogenic protein aggregates, such as mutant huntingtin protein, which causes Huntington's disease, and the $A \beta$ protein, which causes Alzheimer's disease. Among numerous strategies, enhancing the proteasomal degradation of target proteins using proteolysis-targeting chimeric molecules (PROTACs) is a promising approach to lower the levels of disease-causing proteins ${ }^{30,31}$, but proteasomes alone do not efficiency degrade certain large proteins or aggregates due to the narrow cavity of the proteasome, greatly limiting the application of the proteasome in degrading certain large proteins or aggregates. Here, we propose a new strategy for degrading specific target proteins or aggregates that harnesses the power of autophagy, an independent protein-degradation pathway and a bulk degradation system present in all eukaryotic cells in which proteins are engulfed into autophagosomes for subsequent lysosomal degradation. The results of our proof-of-concept experiments suggest a biomimetic strategy that utilizes multifunctional nanoparticles instead of traditional intracellular proteins and mimics their functions. Importantly, we prove the feasibility of surface engineering with high-affinity bound peptides that target pathogenic proteins for degradation via the autophagy pathway. This strategy provides a new platform to target many disease-causing proteins that need to be degraded through a safe and simple nanoparticle system, which is more direct and effective than small-molecule inhibitors. The type of NRs that we have developed provides a new direction for the treatment of diseases caused by protein aggregates, although combatting peptide-targeted protein aggregates need to be further improved and optimized.

\section{Declarations}




\section{Acknowledgement}

This work was supported by grants from the National Natural Science Foundation of China (81771975, $32071398,91859103,52173121)$, Key R\&D Program of Guangdong Province (2020B1515120096, 2020B0101030006), the Natural Science Foundation for Distinguished Young Scholars of Guangdong Province (2018B030306035).

\section{References}

1. Levine, A.J. \& Oren, M. The first 30 years of p53: growing ever more complex. Nat Rev Cancer 9 , 749-758 (2009).

2. Shaw, P.H. The Role of p53 in Cell Cycle Regulation. Pathology - Research and Practice 192, 669675 (1996).

3. Vousden, K.H. \& Lane, D.P. p53 in health and disease. Nat Rev Mol Cell Bio/ 8, 275-283 (2007).

4. Vousden, K.H. \& Ryan, K.M. p53 and metabolism. Nat Rev Cancer 9, 691-700 (2009).

5. Cancer Genome Atlas Research, N. Integrated genomic analyses of ovarian carcinoma. Nature 474, 609-615 (2011).

6. Olivier, M., Hollstein, M. \& Hainaut, P. TP53 mutations in human cancers: origins, consequences, and clinical use. Cold Spring Harb Perspect Biol 2, a001008 (2010).

7. Bertheau, P. et al. p53 in breast cancer subtypes and new insights into response to chemotherapy. Breast 22 Suppl 2, S27-29 (2013).

8. Freed-Pastor, W.A. \& Prives, C. Mutant p53: one name, many proteins. Genes Dev 26, 1268-1286 (2012).

9. $\quad$ Muller, P.A. \& Vousden, K.H. p53 mutations in cancer. Nat Cell Biol 15, 2-8 (2013).

10. Yue, X. et al. Mutant p53 in Cancer: Accumulation, Gain-of-Function, and Therapy. J Mol Bio/ 429, 1595-1606 (2017).

11. Lukashchuk, N. \& Vousden, K.H. Ubiquitination and degradation of mutant p53. Mol Cell Bio/ 27, 8284-8295 (2007).

12. Schulz-Heddergott, R. et al. Therapeutic Ablation of Gain-of-Function Mutant p53 in Colorectal Cancer Inhibits Stat3-Mediated Tumor Growth and Invasion. Cancer Cel/ 34, 298-314 e297 (2018).

13. Zhang, C. et al. Gain-of-function mutant p53 in cancer progression and therapy. J Mol Cell Bio/ 12, 674-687 (2020). 
14. Bykov, V.J.N., Eriksson, S.E., Bianchi, J. \& Wiman, K.G. Targeting mutant p53 for efficient cancer therapy. Nat Rev Cancer 18, 89-102 (2018).

15. Parrales, A. \& Iwakuma, T. Targeting Oncogenic Mutant p53 for Cancer Therapy. Front Onco/ 5, 288 (2015).

16. Kocaturk, N.M. \& Gozuacik, D. Crosstalk Between Mammalian Autophagy and the UbiquitinProteasome System. Front Cell Dev Biol 6, 128 (2018).

17. Jing, M. et al. Photoresponsive PAMAM-Assembled Nanocarrier Loaded with Autophagy Inhibitor for Synergistic Cancer Therapy. Small 17, e2102295 (2021).

18. Lee, C.W. et al. Selective autophagy degrades nuclear pore complexes. Nat Cell Bio/ 22, 159-166 (2020).

19. Zhang, Y. et al. Harnessing copper-palladium alloy tetrapod nanoparticle-induced pro-survival autophagy for optimized photothermal therapy of drug-resistant cancer. Nat Commun 9, 4236 (2018).

20. Khaminets, A., Behl, C. \& Dikic, I. Ubiquitin-Dependent And Independent Signals In Selective Autophagy. Trends Cell Biol 26, 6-16 (2016).

21. Kirkin, V. \& Rogov, V.V. A Diversity of Selective Autophagy Receptors Determines the Specificity of the Autophagy Pathway. Mol Cel/ 76, 268-285 (2019).

22. Shaid, S., Brandts, C.H., Serve, H. \& Dikic, I. Ubiquitination and selective autophagy. Cell Death Differ 20, 21-30 (2013).

23. Sarraf, S.A. et al. Loss of TAX1BP1-Directed Autophagy Results in Protein Aggregate Accumulation in the Brain. Mol Cell 80, 779-795 e710 (2020).

24. Jo, C. et al. Nrf2 reduces levels of phosphorylated tau protein by inducing autophagy adaptor protein NDP52. Nat Commun 5, 3496 (2014).

25. Pankiv, S. et al. p62/SQSTM1 binds directly to Atg8/LC3 to facilitate degradation of ubiquitinated protein aggregates by autophagy. J Biol Chem 282, 24131-24145 (2007).

26. Guida, E. et al. Peptide aptamers targeting mutant p53 induce apoptosis in tumor cells. Cancer Res 68, 6550-6558 (2008).

27. Man, N., Chen, Y., Zheng, F., Zhou, W. \& Wen, L.P. Induction of genuine autophagy by cationic lipids in mammalian cells. Autophagy 6, 449-454 (2010).

28. Roberts, R. et al. Autophagy and formation of tubulovesicular autophagosomes provide a barrier against nonviral gene delivery. Autophagy 9, 667-682 (2013). 
29. Alexandrova, E.M. et al. Improving survival by exploiting tumour dependence on stabilized mutant p53 for treatment. Nature 523, 352-356 (2015).

30. Li, Z. et al. Allele-selective lowering of mutant HTT protein by HTT-LC3 linker compounds. Nature 575, 203-209 (2019).

31. Winter, G. E. , et al. Phthalimide conjugation as a strategy for in vivo target protein degradation. Science 348, 1376-1381(2015).

32. Li, H.-J. et al. Stimuli-responsive clustered nanoparticles for improved tumor penetration and therapeutic efficacy. Proceedings of the National Academy of Sciences of the United States of America 113, 4164-4169 (2016).

\section{Methods}

Regents and antibodies. Cycloheximide (5087390001), MG-132 (474791), wortmannin (W3144), 3methyladenine (M9281), brefeldin A (B5936), CDDP (PHR1624) was purchased from Sigma-Aldrich. LysoTracker Red DND-99 (L7528) and Lipofectamine 3000 (L3000015) were from Invitrogen. Anti-p53 (sc-126, Mouse mAb), anti-ATG5 (sc-133158) and anti-SQSTM1 (sc-28359) antibodies were purchased from Santa Cruz Biotechnology. Anti-ubiquitin (58395S) and anti-K63-polyubiquitin (12930S) antibodies were purchased from Cell Signaling Technology. Anti-LC3 antibody (NB100-2220) was purchased from Novus Biologicals. Anti-GAPDH antibodies (AB9132) was purchased from Chemicon. Goat anti-rabbit (Alexa Fluor 488, A-31566) and goat anti-mouse (Alexa Fluor 647, A-21242) secondary antibodies were from Invitrogen.

Cells. Cell culture reagents were purchased from Gibco (Carlsbad, CA). ES-2 and SK-BR-3 were grown in McCoy's 5A Medium. NCl-H1299 and HUVEC were grown in RPMI 1640 medium. MDA-MB-231, MIA PaCa2 and HEK 293 were grown in DMEM medium. All mediums were supplemented with $10 \%$ fetal bovine serum (Gibco) and 1\% penicillin-streptomycin (Gibco) except for MIA PaCa-2, and MIA PaCa-2 were supplemented with $10 \%$ fetal bovine serum (Gibco), $2.5 \%$ horse serum and $1 \%$ penicillin-streptomycin (Gibco). All cell lines were from ATCC and were cultured at $37^{\circ} \mathrm{C}$ under $5 \% \mathrm{CO}_{2}$.

Preparation of NRs nanoparticles: A mixture containing polymer (Mal-PEG $2000-\mathrm{PLA}_{2000}, 10.0 \mathrm{mg}$ ), DOTAP $(2.0 \mathrm{mg}$ ) was dissolved in $1.2 \mathrm{~mL}$ THF solution and then gradually added to $10 \mathrm{~mL} P B S(\mathrm{pH}=7.4,0.01 \mathrm{M})$ with stirring. After additional stirring for $2 \mathrm{~h}$, the nanoparticles solution evaporated by rotary evaporator to remove THF for $2 \mathrm{~h}$, and obtained nanoparticles denoted as dNRs. Then, MBP ( $5 \mathrm{mg}, 0.0025 \mathrm{mmol})$ was added into the $\mathrm{mNRs}$ solution and sustained stirring for $8 \mathrm{~h}(\mathrm{pH}=7.4,0.01 \mathrm{M})$. Then, the solution was transferred into a dialysis bag (MWCO $15000 \mathrm{Da})$ to remove free MBP against PBS $(2.0 \mathrm{~L}, \mathrm{pH}=7.4,0.01$ M) for $12 \mathrm{~h}$. After ultrafiltration and concentration using Amicon YM-30 centrifugal filter devices (Millipore, MWCO $5000 \mathrm{Da}$ ), the obtained nanoparticles denoted as NRs. mNRs were also prepared by a similar method without added DOTAP during the preparation process. 
Preparation of Pt(IV)-loaded NRs nanoparticles: Firstly, the Pt(IV) prodrugs were synthsiszed by previous method $^{32}$ Then, a mixture containing polymer (Mal-PEG $\left.2000-\mathrm{PLA}_{2000}, 10.0 \mathrm{mg}\right)$, DOTAP $(2.0 \mathrm{mg})$ and $\mathrm{Pt}(\mathrm{IV})$ prodrug $(1.5 \mathrm{mg})$ was dissolved in $1.4 \mathrm{~mL}$ THF solution and then gradually added to $10 \mathrm{~mL}$ PBS $(\mathrm{pH}=7.4,0.01 \mathrm{M})$ with stirring. After additional stirring for $2 \mathrm{~h}$, the nanoparticles solution evaporated by rotary evaporator to remove THF for $2 \mathrm{~h}$, and obtained nanoparticles denoted as dNRs/Pt(IV). Then, MBP ( $5 \mathrm{mg}, 0.0025 \mathrm{mmol}$ ) was added into the $\mathrm{dNRs} / \mathrm{Pt}(\mathrm{IV})$ solution and sustained stirring for $8 \mathrm{~h}(\mathrm{pH}=7.4,0.01$ $\mathrm{M})$. Then, the solution was transferred into a dialysis bag (MWCO $14000 \mathrm{Da})$ to remove free MBP and free Pt(IV) prodrug against PBS $(2.0 \mathrm{~L}, \mathrm{pH}=7.4,0.01 \mathrm{M})$ for $12 \mathrm{~h}$. After ultrafiltration and concentration using Amicon YM-30 centrifugal filter devices (Millipore, MWCO $5000 \mathrm{Da}$ ), the obtained nanoparticles denoted as NRs/Pt(IV). dNRs/Pt(IV) were also prepared by a similar method without added MBP during the preparation process.

Characteristic of these nanoparticles: The zeta potential and size distribution of mNRs, dNRs, and NRs measured by Malvern ZS90 dynamic light scattering (DLS) instrument (Malvern Instruments Ltd., England). The nanoparticles mNRs, dNRs and NRs were dispersed in pH 7.4 PBS (0.01 M) containing 10\% FBS at the concentration of $1.0 \mathrm{mg} / \mathrm{mL}$ and incubated at $37^{\circ} \mathrm{C}$. Then, the samples were taken at a designated time point, and the diameter of NPs was measured by Malvern ZS90 dynamic light scattering (DLS) instrument (Malvern Instruments Ltd., England). Then, $10 \mu \mathrm{L}$ mNRs, dNRs or NRs were added dropwise to copper gauze with a carbon film (400 mesh) and dried overnight, respectively. The morphology and aggregation of the mixed particles were observed by TEM. To confirm that the MBP was bonding to the NRs surface and the Mutp53 could immobilize onto NRs via specific recognition. The NRs core was labeled with Rhodamine B (RhoB) by incorporated RhoB-PEG- $b$-PLA during the NPs preparation process, then the Aleax fluor ${ }^{\mathrm{TM}} 488$ (Succinimidyl ester) labeled MBP was added and reacted with NPs through click reaction. After that, the Aleax fluor ${ }^{\mathrm{TM}} 647$ (Succinimidyl ester) labeled Mutp53 protein was added to the above bifluorescence labeled NRs and continue stirring for $4 \mathrm{~h}$. Then, the fluorescencelabeled NRs or mixtures (NRs plus Mutp53) were dropped on the coverslip and precipitated for $10 \mathrm{~min}$, and images were acquired using an Abbelight STORM Super-resolution System (Quantum Design China, Ltd.), which is equipped with $488 \mathrm{~nm}, 530 \mathrm{~nm}$ and $647 \mathrm{~nm}$ super-resolution compatible laser excitation, enables industry-leading multi-color simultaneous STORM acquisition.

Circular Dichroism Spectroscopy: Ultraviolet CD spectra were obtained using a Chirascan CDspectrometer with a $1 \mathrm{~mm}$ path length cuvette (Applied photophysics limited, England). For the measurement of the combination, the previously prepared mutp53 protein stock solution was diluted to concentrations of $1.25 \mathrm{mM}$ and mixed with $\mathrm{mNRs}$, dNRs and NRs ([NPs] $=5 \mathrm{mg} / \mathrm{mL}$, [MBP] $=1.25 \mathrm{mM}$ ) in a volume ratio of $1 / 1$. Spectra of solutions with and without protein were recorded between 195 and 260 $\mathrm{nm}$ at $65^{\circ} \mathrm{C}$. The free mutp53 protein and MBP plus mutp53 protein were used as a control group.

Surface plasmon resonance: The GE BIAcore $8 \mathrm{~K}$ instrument was operated at a constant temperature of $25^{\circ} \mathrm{C}$ and the $\mathrm{CM} 5$ sensor chip (GE Healthcare) was used in this study. Each CM5 sensor chip consists of 8 identical experimental channels and each channel was divided into two flow cells. In our experimental setup, flow-cell 1 (Fc1) was always kept blank as a reference, while flow-cell 2 (Fc2) was functionalized 
with the nanocarrier for interaction studies with mutant p53 protein. Specifically, the system was first equilibrated with PBS-T buffer (20 mM Na-phosphate, $150 \mathrm{mM} \mathrm{NaCl}$, and $0.05 \%$ Tween 20, pH 7.4). One experiment channel was activated for 6 min with a mixture of EDC $(0.2 \mathrm{M})$ and NHS (0.05 M). In Fc2, this was followed by $7 \mathrm{~min}$ injection of dNRs in $10 \mathrm{mM}$ acetate buffer ( $\mathrm{pH}$ 4.0). In parallel, PBS-T buffer was injected in Fc2. Finally, $1 \mathrm{M}$ ethanolamine- $\mathrm{HCl}$ solution was injected onto both $\mathrm{Fc} 1$ and $\mathrm{Fc} 2$ to block the remaining NHS-ester groups. To observe the interactions between mutp53 and NRs, $5.21 \mu \mathrm{M}$ mutp53 in PBS solution was injected into the nanocarrier modified sensor surface. The injection time is $3 \mathrm{~min}$ at a flow rate of $30 \mu \mathrm{l} / \mathrm{min}$, followed by a dissociation step of $5 \mathrm{~min}$. To calculate the KD value, a new channel was prepared using identical conditions. 5 different concentrations $(4 \mathrm{nM}, 20 \mathrm{nM}, 100 \mathrm{nM}, 500 \mathrm{nM}$ and $2500 \mathrm{nM}$ ) of mutp53 in PBS solution were injected onto the channel with the response units measured at single cycle. All signals were subtracted from the reference signal. The binding kinetics was calculated using a 1:1 binding model with BIAcore 8K Evaluation software. The interactions between mutp53 and dNRs were measured as a control group.

Electron microscopy. After harvesting, ES-2 cells were fixed in suspension with $4 \%$ glutaraldehyde in 0.1 Mcalcodyate buffer ( $\mathrm{pH} 7.3$ ) overnight at $4^{\circ} \mathrm{C}$. Then, cells were post-fixed in $2 \%$ osmium tetroxide in $0.1 \mathrm{M}$ osmium acid for 1 hour at room temperature. After dehydration with gradient ethanol and propylene oxide, the cells were embedded in epoxy resin, and then the area containing the cells was cut into ultrathin sections, stained with uranyl acetate and lead citrate, and observed under a transmission electron microscope (JEOL-1230, Japan).

Establishment of EGFP-LC3/MDA-MB-231 cells. For establishing EGFP-LC3/MDA-MB-231 cells, MDA-MB231 cells were transfected with pEGFP-LC3 using Lipofectamine 3000 (Invitrogen, USA) according to the manufacturer's protocol. $24 \mathrm{~h}$ later transfection cells were transferred to a new plate and underwent selection in DMEM medium containing $0.6 \mathrm{mg} / \mathrm{ml}$ of G418 (Promega, USA). About 10 days posttransfection and expansion, cell colonies exhibiting strong green fluorescence were selected under a fluorescence microscope.

Immunofluorescence. Cells cultured on coverslips were fixed with $4 \%$ paraformaldehyde for 15 min and then permeabilized with $0.1 \%$ Triton X-100 in PBS for 15 min, followed by blocking in $1 \%$ BSA for $1 \mathrm{~h}$. After an overnight incubation with the primary antibodies at $4^{\circ} \mathrm{C}$, the coverslips were washed three times with PBS and incubated with fluorescent secondary antibodies (Invitrogen, 1:2000) for $1 \mathrm{~h}$ in the dark at room temperature. The coverslips were then washed three times in PBS and stain the nucleus with DAPI (Roche). Images were acquired using a confocal microscope (Nikon, Ti-E A1). For immunofluorescence of paraffin-embedded tissues, the slices were dewaxed and rehydrated in xylene and graded alcohol solutions first, followed by the steps of normal immunofluorescence. For detecting p53 degradation, antip53 antibody (sc-126, 1:100 dilution) and goat anti-mouse (Alexa Fluor 647, A-21242,1:2000 dilution) secondary antibody were used.

Western blotting. Cells were harvested and lysed with radoimmunoprecipitation assay (RIPA) buffer containing protease inhibitor cocktail (C600386, Sangon) on ice. An equal volume of $2 \times$ SDS sample 
buffer was added, and boiled for $10 \mathrm{~min}$. Cell lysate containing 20-100 $\mu \mathrm{g}$ proteins were separated by electrophoresis on a SDS polyacrylamide gel and transferred to PVDF membrane (Millipore, IPVH00010). After blocking with $5 \%$ nonfat dry milk for $1 \mathrm{~h}$, the PVDF membrane was incubated $3 \mathrm{~h}$ with a primary antibody against specific proteins at room temperature, extensively washed with TBST and incubated with horseradish peroxidase-conjugated secondary antibody (1:10,000 dilution) for $1 \mathrm{~h}$, and finally visualized with an enhanced chemiluminescence (ECL) kit and imaged by the GE Amersham Imager 600 imaging system.

Cell viability assay. Cells were grown in 96-well plates at a density of approximately 10,000 cells per well. Twenty-four hours later, cells were exposed to indicated treatment for $24 \mathrm{~h}$, followed by MTT assay. Briefly, MTT (thiazoyl blue tetrazolium bromide, Bio Basic) was added to the growing cultures at a final concentration of $0.5 \mathrm{mg} / \mathrm{mL}$ and incubated for $4 \mathrm{~h}$ at $37^{\circ} \mathrm{C}$. Then, the medium was replaced with DMSO to dissolve blue formazan crystals, and plates were shaken for $15 \mathrm{~min}$ in the dark. Results were measured with a spectrophotometer (Elx800, BioTek, Winooski, VT, USA) at $570 \mathrm{~nm}$.

Quantitative PCR analysis. The mRNA expression level of mutp53 was measured by q-PCR. According to the manufacturer's instructions, total RNA from ES-2 cells was extracted using AG RNAex Pro Reagent (AG21101), and purified total RNA was reverse-transcribed to cDNA using the Evo M-MLV RT Kit (AG11711). Then, $2 \mu \mathrm{L}$ of cDNA was used as template in subsequent quantitative PCR reactions conducted on an ABI7300 using SYBR Green Super Mix kit (Roche). The primers were P53-F (GCGTGAGCGCTTCGAGAT), P53-R (AGCCTGGGCATCCTTGAG), GAPDH-F (GTCGTACCACAGGCATTGTGATGG), and GAPDH-R (GCAATGCCTGGGTACATGGTGG).

Plasmid construction and transfection. All p53 mutations (S241F, R248W, R175H) were generated by QuickMutation site-directed mutagenesis kit based on pcDNA3.1-p53 plasmid according to the product manual (D0206, Beyotime). All plasmids were sequenced to confirm the designed mutation. For transfection of plasmid DNA, cells were grown to $70-80 \%$ confluency and transfected with plasmid DNA using Lipofectamine 3000 (Invitrogen, USA) according to the manufacturer's instructions. After 24 hours, the transfected cells were transferred to a new dish and selected in DMEM medium containing $0.6 \mathrm{mg} / \mathrm{ml}$ G418 (Promega, USA). About 10 days post-transfection and expansion, the selected cells were used for subsequent experiments.

Gene knockdown. PLKO vector (Sigma-Aldrich) was co-transfected with plasmids encoding gag and vsvg into HEK 293T packaging cells using lipofectamine 3000 (Invitrogen). Then, after 48-hour transfection, viral supernatant was collected and added into the culture medium of MDA-MB-231 cells in the presence of $5 \mu \mathrm{g} / \mathrm{mL}$ polybrene (Sigma-Aldrich). The infected cells were selected by puromycin to establish stable cells. shATG5 in PLKO vector: 5'-CCAGATATTCTGGAATGGAAA-3'.

p53 ubiquitination studies. ES-2 cells after NRs treatment were harvested and lysed with RIPA buffer containing protease inhibitor cocktail (Sangon). Cell extracts containing an equal amount of protein were incubated with Protein A/G-Agarose (sc-2003) and p53 antibody (sc-126, $2 \mu \mathrm{g}$ per sample) overnight. After washing with PBST buffer, the precipitants were resolved on SDS-PAGE for western blotting with 
antibodies against ubiquitin (58395S, 1:1000 dilution), K63-Ub (12930S, 1:1000 dilution) or p53 (ab32389, 1:5000 dilution).

Sphere-formation assay. Single-cell suspensions of ES-2 cells were plated (1,000 cells per well) into 24well Ultra Low Attachment plates (Corning) in serum-free RPMI 1640 culture media (Gibco) supplemented with $2 \%$ B27 (Invitrogen), basic fibroblast growth factor $(20 \mathrm{ng} / \mathrm{ml}$, Invitrogen) and epidermal growth factor $\left(20 \mathrm{ng} / \mathrm{ml}\right.$, Millipore). The cells were grown in humidified atmosphere with $5 \% \mathrm{CO}_{2}$ for 7 days, the half of old culture medium was replaced by an equal amount of fresh culture medium every 3 day. The diameters of spheres in different groups were recorded with inverted phase-contrast microscopy (Nikon, DS-Fi3).

Transwell assay. RPMI 1640 media supplemented with 10\% FBS was added to the lower chamber. ES-2 cells $\left(1 \times 10^{4}\right.$ per well) were added to the upper chambers in serum-free 1640 . After 12 hours incubation with PBS, free peptide, mNRs, dNRs, NRs, cells which migrated to the lower face of the membrane were fixed with methanol and stained with $0.1 \%$ of crystal violet. After being washed by PBS for 3 times, cells were imaged with microscopy (Nikon, Ci-L).

Colony formation assay. $24 \mathrm{~h}$ after seeding ES-2 cells (500 per well) on a 6-well plate, cells were treated with PBS, free peptide, mNRs, dNRs, NRs for 24 hours and then changed to new medium. After culturing for another 10 days, colonies were fixed with methanol, stained with $0.1 \%$ of crystal violet and imaged.

Hoechst/PI double staining. Briefly, ES-2 cells at $5 \times 10^{3}$ cells/well in 96 -well plates were incubated with nanoparticles for $24 \mathrm{~h}$. After treatment, cellular nuclei were stained with Hoechst $33342(10 \mu \mathrm{g} / \mathrm{mL})$. Propidium lodide $(10 \mu \mathrm{g} / \mathrm{mL}$ ) was used to observe cell death by fluorescence microscopy (Nikon, DS-Fi3). Cell death was expressed by the ratio of PI-positive cells to Hoechst positive cells.

CRISPR/Cas9 System. CRISPR/Cas9 system was used to partially knock out p53 in ES-2 cells. Single guide RNA (sgRNA) is generated by the online CRISPR design tool (http://crispr.mit.edu/). The sgRNA sequences were cloned into PX458 gene vector, and transfected into ES-2 cells with Lipofectamine 3000 (Invitrogen, L3000015) in Opti-MEM according to the manufacturer's instructions. The p53-specific gRNA sequence was: sgp53-F:5-CACCGCCATTGTTCAATATCGTCCG, sgp53-R:5AAACCGGACGATATTGAACAATGGC;

Animals. BALB/c mice (female, 18-20 g, 5-6 weeks), BALB/c nude mice (female, 18-20 g, 5-6 weeks) and NOD/SCID mice (female, 18-20 g, 5-6 weeks) were purchased from Beijing Vital River Laboratory Animal Technology Co., Ltd. (Beijing, China). All animals received care in compliance with the guidelines outlined in the Guide for the Care and Use of Laboratory Animals and maintained at the SPF animal facility at the South China University of Technology (SCUT).

Toxicity study. BALB/c mice were randomly divided into five groups (5 mice per group) and injected intravenously with $25 \mathrm{mg}$ per $\mathrm{kg}$ of dNRs, dNRs/Pt, NRs or NRs/Pt or PBS. $24 \mathrm{~h}$ after injection, the blood serum was collected and mouse alanine aminotransferase (ALT), aspartate transaminase (AST), blood 
urea nitrogen (BUN) and serum creatinine (SCR) were measured using Automatic Biochemical Analyzer (3100, Hitachi, Japan) according to the manufacturer's instructions. The major organs of the mice after treatment were also collected and stained by haematoxylin \& eosin (H\&E).

In vivo biodistribution. DiD-labelled NRs was prepared by label DiD with NRs. The real-time imaging of DiD-NRs ( $25 \mathrm{mg} / \mathrm{kg}$ ) was conducted on ES-2 tumor-bearing BABL/c nude mice using the In-Vivo Xtreme (Bruker, German). After $24 \mathrm{~h}$, mice were sacrificed and major organs (heart, liver, spleen, lung, kidney, and tumor) were harvested from mice and imaged in vitro.

Animal model studies. For the ES-2 tumor model, $1 \times 10^{7}$ of ES- 2 cells were subcutaneously injected in the right flank of the BALB/c nude mice. Mice were randomly divided into seven groups ( 6 mice for each group), and when tumor volumes reached about $100 \mathrm{~mm}^{3}$, the treatment procedures were initiated, with mice receiving the indicated injection into the tail vein every 2 days. The respective treatments were: PBS, $\operatorname{CDDP}(1 \mathrm{mg} / \mathrm{kg}), \mathrm{dNRs}(25 \mathrm{mg} / \mathrm{kg}), \mathrm{dNRs} / \mathrm{Pt}(25 \mathrm{mg} / \mathrm{kg})$, NRs $(25 \mathrm{mg} / \mathrm{kg}), \mathrm{NRs} / \mathrm{Pt}$ ( $25 \mathrm{mg} / \mathrm{kg})$. Treatment was terminated on day 13 , with the mice sacrificed and the excised tumors photographed and weighed. For the PDX tumor model, a human ovarian carcinoma specimen was obtained from Nanfang Hospital

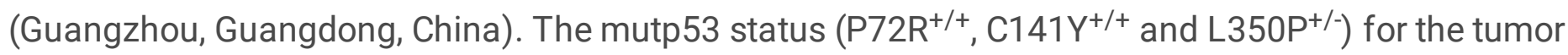
was verified by whole-exome sequencing conducted at Beijing Genomics Institute as well as by sequencing of the full-length p53 coding sequence after RT-PCR. The experiments using patient-derived materials have complied with all relevant ethical regulations and were performed according to the approved guidelines established by the Institutional Human Research Subjects Protection Committee of the Ethics Committee of the South China University of Technology, with the informed consent from the patients. The tumor tissue obtained after surgery was cut into $2 \sim 3 \mathrm{~mm}^{3}$ fragments and orthotopically explanted in the mammary fat pad of the NOD/SCID mice. Once reaching approximately $800 \mathrm{~mm}^{3}$, tumor xenografts were cut into $2 \sim 3 \mathrm{~mm}^{3}$ slices and propagated by following the same explanting procedure. The mice bearing xenografts of the third generation were randomly divided into 4 groups containing 6 mice per group and received the indicated treatments when tumor grew to about $100 \mathrm{~mm}^{3}$. Treatment lasted 13 days, following the same procedure as described for the ES-2 model. The body weight and tumor sizes of mice was measured every 2 days, and tumor volume was calculated by the formula $V=I w^{2}$ $2^{-1}$, in which I and $w$ indicate the length and width of the tumor, respectively.

TUNEL assay. ES-2 and PDX tumors that received various treatments were used for TUNEL assay (Beyotime, C1088). $4 \mathrm{~mm}$ frozen sections were washed with PBS and then incubated with proteinase $\mathrm{K}$ for 20 min at room temperature. After washing 3 times with PBS to remove unreacted protease $K$, the TUNEL reaction mixture was added to rinsed slides and incubated in a humidified chamber for 60 min at $37^{\circ} \mathrm{C}$. After washing with PBS, the sections were stained for 10 min with DAPI, washed with PBS, and visualized with a fluorescence microscope (Nikon, DS-Fi3)

Immunohistochemistry $(\mathrm{IHC})$. The slices of paraffin-embedded tissues were dewaxed and rehydrated in xylene and graded alcohol solutions. Anti-p53 antibody (sc-126, 1:200 dilution) were used to stain the 
slices. Cell nuclei were stained with hematoxylin.

Statistical analysis. All data were expressed as Mean \pm SEM or Mean \pm SD, and analyzed by ANOVA. *P < $0.05,{ }^{*} \mathrm{P}<0.01$ and ${ }^{* \star *} \mathrm{P}<0.001$ were considered statistically significant.

\section{Figures}

\section{Figure 1}

Preparation of engineered biomimetic NRs to mimic autophagy receptors. (a) Schematic of the preparation of NRs and their ability to bind mutant p53 (mutp53) proteins. (b) TEM image of dNRs, mNRs and NRs. Scale bar: 200 nm. (c) Circular dichroism (CD) measurements of mutp53 mixed with different formulations (1 x PBS). (d) The interaction between mutp53 and NRs was measured by surface plasmon resonance (SPR). (e) (f) Stochastic optical reconstruction microscopy (STORM) images of NRs (e) and NRs that have captured mutp53 (f). PEG-PLA NP: red (RhoB), mutp53-binding peptides: green (Alexa Fluor 488) and mutp53 protein: blue (Alexa Fluor 647). Scale bar: $100 \mathrm{~nm}$.

\section{Figure 2}

NRs induce the rapid degradation of mutp53. (a) Western blotting of p53 and GAPDH in ES-2 cells after treatment with NRs $(500 \mu \mathrm{g} / \mathrm{mL})$ and different concentrations of DOTAP. (b) Confocal microscopic images of ES-2 cells treated with PBS (Cont), peptide $(250 \mu \mathrm{g} / \mathrm{mL}), \mathrm{dNRs}(500 \mu \mathrm{g} / \mathrm{mL}), \mathrm{mNRs}(500$ $\mu \mathrm{g} / \mathrm{mL})$ or NRs $(500 \mu \mathrm{g} / \mathrm{mL})$ for $12 \mathrm{~h}$, followed by immunostaining with anti-p53 antibody and nuclear staining with DAPI. Scale bar, $10 \mu \mathrm{m}$. (c) Western blotting of p53, LC3 and GAPDH in ES-2 cells treated with PBS (Cont), peptide $(250 \mu \mathrm{g} / \mathrm{mL})$, mNRs $(500 \mu \mathrm{g} / \mathrm{mL})$, dNRs $(500 \mu \mathrm{g} / \mathrm{mL})$ or NRs $(500 \mu \mathrm{g} / \mathrm{mL})$ for 12 h. GAPDH served as a loading control. (d) Relative p53 mRNA levels in ES-2 cells treated with PBS (Cont) or $500 \mu \mathrm{g} / \mathrm{mL}$ NRs for $12 \mathrm{~h}$, as assayed by quantitative RT-PCR. The values for the NRs group were normalized against those for the Cont group. Mean \pm SEM. $n=3$. Student's t-test. (e) Western blotting of p53 and GAPDH in ES-2 cells treated with cycloheximide (CHX, $20 \mu \mathrm{M})$ in the absence or presence of NRs $(500 \mu \mathrm{g} / \mathrm{mL})$ for the indicated time in hours. (f) Western blotting of p53, LC3 and GAPDH in ES-2 and MDA-MB-231 cells treated with 0, 250 or $500 \mu \mathrm{g} / \mathrm{mL}$ NRs for $12 \mathrm{~h}$. (g) Western blotting of p53, LC3 and GAPDH in H1299 cells transfected with p53S241F, p53R248W or p53R175H plasmids for 24 h, followed by treatment with PBS (Cont) or NRs $(500 \mu \mathrm{g} / \mathrm{mL})$ for $12 \mathrm{~h}$.

\section{Figure 3}

NRs cause the autophagy-dependent degradation of mutp53 proteins. (a) Western blotting of p53 and GAPDH in ES-2 cells after the indicated treatment for $12 \mathrm{~h}$. Concentrations used: NRs, $500 \mu \mathrm{g} / \mathrm{mL}$; 
wortmannin, $1 \mu \mathrm{M}$; MG-132, $10 \mu \mathrm{M}$. (b) Western blotting of ATG5, p53, LC3 and GAPDH in ES-2 cells treated with $500 \mu \mathrm{g} / \mathrm{mL}$ NRs for $12 \mathrm{~h}$ after transfection with ATG5 shRNA (sh ATG5) or negative control shRNA (sh NC) for 48 h. (c) TEM analysis of ES-2 cells treated with PBS (Cont) or $500 \mu \mathrm{g} / \mathrm{mL}$ NRs for 12 $\mathrm{h}$. The right panel is a high-magnification image of the indicated portion. Arrows indicate autophagosomes and autolysosomes. Scale bar: $500 \mathrm{~nm}$. (d) Confocal microscopic images of EGFPLC3/MDA-MB-231 cells treated with PBS (Cont) or $500 \mu \mathrm{g} / \mathrm{mL}$ NRs for $12 \mathrm{~h}$. Scale bar, $10 \mu \mathrm{m}$. (e) Western blotting of p53, LC3 and GAPDH after ES-2 cells were subjected to the indicated treatment for $12 \mathrm{~h}$. Concentrations used: NRs, $500 \mu \mathrm{g} / \mathrm{mL}$; 3-MA, $5 \mathrm{mM}$; bafilomycin A1 (BFA), $400 \mathrm{nM}$. (f) Western blotting of SQSTM1, LC3 and GAPDH in ES-2 cells after treatment with NRs $(500 \mu \mathrm{g} / \mathrm{mL})$ for various times. $(\mathrm{g})$ Confocal microscopic images of MDA-MB-231-EGFP-Mcherry-LC3 cells treated with mNRs $(500 \mu \mathrm{g} / \mathrm{mL})$ or NRs $(500 \mu \mathrm{g} / \mathrm{mL})$ for $12 \mathrm{~h}$. Scale bar: $20 \mu \mathrm{m}$. (h) Western blotting of p53, LC3 and GAPDH in ES-2 cells after the indicated treatments for $12 \mathrm{~h}$. Dosing: NRs, $500 \mu \mathrm{g} / \mathrm{mL}$; PYR-41, $20 \mu \mathrm{M}$. (i) ES-2 cells were treated with PBS or NRs $(500 \mu \mathrm{g} / \mathrm{mL})$ for $12 \mathrm{~h}$. Whole-cell extracts were immunoprecipitated with anti-p53 antibody and then immunoblotted with anti-K63-Ub and anti-Ub antibodies. The lower panel shows p53 Western blotting of the immunoprecipitated proteins.

\section{Figure 4}

Degradation of mutp53 by NRs abrogated mutp53-conferred GOF phenotypes. (a) Cell viability of normal cells (HEK 293T cells and HUVECs) and mutp53-expressing tumor cells (MIA PaCa-2, SK-BR-3, MDA-MB231 and ES-2 cells) treated with PBS or NRs $(500 \mu \mathrm{g} / \mathrm{mL})$ for $24 \mathrm{~h}$. Mean \pm SEM. $\mathrm{n}=4$, Student's t-test. (b) Cell viability of ES-2 cells after treatment with PBS (Cont) or NRs $(250 \mu \mathrm{g} / \mathrm{mL})$ for $24 \mathrm{~h}$ following transfection with the p53-specific Cas9 gRNA (Cas9 p53) or negative control Cas9 gRNA (Cas9 Cont) for 24 h. Mean \pm SEM. $n=3$, Student's t-test. (c) Sphere-formation behavior of ES-2 cells. After the indicated treatments for $12 \mathrm{~h}$, cells were cultured for 7 days and then photographed with a microscope. Concentrations used: peptide $(250 \mu \mathrm{g} / \mathrm{mL})$, mNRs (500 $\mu \mathrm{g} / \mathrm{mL})$, dNRs $(500 \mu \mathrm{g} / \mathrm{mL})$, NRs $(500 \mu \mathrm{g} / \mathrm{mL})$. (d) Colony-formation behavior of ES-2 cells. After treatment for $12 \mathrm{~h}$ with PBS (Cont), peptide $(250 \mu \mathrm{g} / \mathrm{mL})$, $\mathrm{mNRs}(500 \mu \mathrm{g} / \mathrm{mL}), \mathrm{dNRs}(500 \mu \mathrm{g} / \mathrm{mL})$ or NRs $(500 \mu \mathrm{g} / \mathrm{mL})$, cells were cultured for 10 days, stained with $0.1 \%$ crystal violet and photographed. (e) Transwell assay of ES-2 cells after treatment with PBS (Cont), peptide $(250 \mu \mathrm{g} / \mathrm{mL}), \mathrm{mNRs}(500 \mu \mathrm{g} / \mathrm{mL})$, dNRs $(500 \mu \mathrm{g} / \mathrm{mL})$ or NRs $(500 \mu \mathrm{g} / \mathrm{mL})$, followed by staining with $0.1 \%$ crystal violet and viewing with a microscope. (f) PI staining (red) of ES-2 cells after treatment with PBS (Cont), peptide $(250 \mu \mathrm{g} / \mathrm{mL})$, mNRs $(500 \mu \mathrm{g} / \mathrm{mL})$, dNRs $(500 \mu \mathrm{g} / \mathrm{mL})$ or NRs $(500 \mu \mathrm{g} / \mathrm{mL})$ for 24 h. Nuclei were stained with DAPI (blue). Scale bar, $100 \mu \mathrm{m}$. (g) Cell viability of ES-2 cells after treatment with PBS, CDDP $(10 \mu \mathrm{g} / \mathrm{mL})$, NRs $(250 \mu \mathrm{g} / \mathrm{mL})$ or NRs $(250 \mu \mathrm{g} / \mathrm{mL})$ combined with CDDP for $24 \mathrm{~h}$. Mean \pm SEM. $n=3$. Student's t-test.

\section{Figure 5}


NRs significantly enhanced chemotherapeutic efficacy in the ES-2 ovarian cancer model. (a) Schematic of the NR/Pt preparation process. (b) In vivo imaging of ES-2 tumor-bearing mice injected with DiDlabeled NRs/Pt via the tail vein at various times. (c) and (d) Fluorescence imaging (c) and quantified results (d) showing NRs in various organs from the mice. Mean \pm SD. $n=3 .{ }^{\star} p<0.05,{ }^{*} p<0.01$. Student's t-test. (e) Changes in tumor volume among mice in the various treatment groups over the 13day therapeutic period. Doses used: $25 \mathrm{mg} / \mathrm{kg}$ dNRs, dNRs/Pt, NRs and NRs/Pt and $1 \mathrm{mg} / \mathrm{kg}$ CDDP. IV injection every two days. Mean \pm SD. $n=5$ or $6 .{ }^{* \star} \mathrm{p}<0.001$. Student's t-test. (f) and (g) Photographs of excised tumors $(\mathrm{f})$ and tumor weight data $(\mathrm{g})$ for the various treatment groups from the ES-2 model on day 13. Mean \pm SD. $n=5$ or $6 .{ }^{* \star *} p<0.001$. Student's t-test. (h) Western blotting of $p 53$ and GAPDH in excised tumor tissues in the various treatment groups from the ES-2 model. (i) Immunohistochemistry of p53 (dark brown staining) in the excised tumor tissues from the various treatment groups from the ES-2 model. Scale bar, $50 \mu \mathrm{m}$.

\section{Figure 6}

NRs significantly enhanced chemotherapeutic efficacy in a patient-derived xenograft (PDX) ovarian cancer model. (a) The 13-day treatment regimen over which the mice received PBS, CDDP, NRs or NRs/Pt by tail vein injection once every two days. (b) The body weights of the various PDX model treatment groups over the 13-day therapeutic period. Dosing: $25 \mathrm{mg} / \mathrm{kg}$ NRs and NRs/Pt, $1 \mathrm{mg} / \mathrm{kg}$ CDDP. IV injection every two days. Mean \pm SD. $n=6$. ${ }^{* \star} p<0.001$. Student's t-test. (c) Changes in tumor volume among the various PDX model treatment groups over the 13-day therapeutic period. Mean \pm SD. $n=6 .{ }^{* \star *}<<0.001$. Student's t-test. (d) Tumor weights in the various PDX model treatment groups on day 13. Mean \pm SD. $n=6$. ${ }^{* \star} p<0.001$. Student's t-test. (e) Western blotting of $p 53$, LC3 and GAPDH in each of the excised tumor tissues from the various PDX model treatment groups. (f) Immunohistochemistry of p53 (top panel) and TUNEL assay (lower panel) in the excised tumor tissues from the various PDX model treatment groups. Scale bar, $50 \mu \mathrm{m}$.

\section{Supplementary Files}

This is a list of supplementary files associated with this preprint. Click to download.

- Supplementarymaterials.pdf 